\title{
Fixed point of asymptotic pointwise nonexpansive semigroups in metric spaces
}

\author{
Saleh Abdullah Al-Mezel ${ }^{1 *}$ and Mohamed Amine Khamsi'i,3
}

${ }^{*}$ Correspondence:

salmezel@kau.edu.sa

${ }^{1}$ Department of Mathematics, King Abdulaziz University, P.O. Box 80203

Jeddah, 21593, Saudi Arabia

Full list of author information is

available at the end of the article

\begin{abstract}
Let $C$ be a bounded, closed, convex subset of a uniformly convex metric space $(M, d)$. In this paper, we introduce the concept of asymptotic pointwise nonexpansive semigroups of nonlinear mappings $T_{t}: C \rightarrow C$, i.e., a family such that $T_{0}(x)=x$, $T_{s+t}=T_{s}\left(T_{t}(x)\right)$, and $d\left(T_{t}(x), T_{t}(y)\right) \leq \alpha_{t}(x) d(x, y)$, where limsup $\sin _{t \rightarrow \infty} \alpha_{t}(x) \leq 1$ for every $x \in C$. Then we investigate the existence of common fixed points for asymptotic pointwise nonexpansive semigroups. The proof is based on the concept of types extended to one parameter family of points.
\end{abstract}

MSC: Primary 47H09; secondary 46B20; 47H10; 47E10

Keywords: fixed point; hyperbolic metric space; inequality; nearest point projection; Mann process; nonexpansive mapping; semigroup; uniformly convex metric space; uniformly Lipschitzian mapping

\section{Introduction}

The purpose of this paper is to prove the existence of common fixed points for semigroups of nonlinear mappings acting in metric spaces. Recently, Khamsi and Kozlowski presented a series of fixed point results for pointwise contractions, asymptotic pointwise contractions, pointwise nonexpansive and asymptotic pointwise nonexpansive mappings acting in modular functions spaces $[1,2]$.

Let us recall that a family $\left\{T_{t}\right\}_{t \geq 0}$ of mappings forms a semigroup if $T_{0}(x)=x$, and $T_{s+t}=$ $T_{s} \circ T_{t}$. Such a situation is quite typical in mathematics and applications. For instance, in the theory of dynamical systems, the vector function space would define the state space, and the mapping $(t, x) \rightarrow T_{t}(x)$ would represent the evolution function of a dynamical system. The question about the existence of common fixed points, and about the structure of the set of common fixed points, can be interpreted as a question whether there exist points that are fixed during the state space transformation $T_{t}$ at any given point of time $t$, and if yes - what does the structure of a set of such points may look like. In the setting of this paper, the state space is a nonlinear metric space.

The existence of common fixed points for families of contractions and nonexpansive mappings in Banach spaces has been the subject of the intensive research since the early 1960s, as investigated by Belluce and Kirk [3, 4], Browder [5], Bruck [6], DeMarr [7], and Lim [8]. The asymptotic approach for finding common fixed points of semigroups of Lipschitzian (but not pointwise Lipschitzian) mappings has also been investigated, see, e.g., Tan and $\mathrm{Xu}$ [9]. It is worthwhile mentioning the recent studies on the special case, when the parameter set for the semigroup is equal to $\{0,1,2,3, \ldots\}$, and $T_{n}=T^{n}$, the $n$th iterate 
of an asymptotic pointwise nonexpansive mapping. Kirk and Xu [10] proved the existence of fixed points for asymptotic pointwise contractions and asymptotic pointwise nonexpansive mappings in Banach spaces, while Hussain and Khamsi [11] extended this result to metric spaces, and Khamsi and Kozlowski to modular function spaces [1, 2]. In the context of modular function spaces, Khamsi discussed in [12] the existence of nonlinear semigroups in Musielak-Orlicz spaces and considered some applications to differential equations.

\section{Uniform convexity in metric spaces}

Throughout this paper, $(M, d)$ will stand for a metric space. Suppose that there exists a family $\mathcal{F}$ of metric segments such that any two points $x, y$ in $M$ are endpoints of a unique metric segment $[x, y] \in \mathcal{F}([x, y]$ is an isometric image of the real line interval $[0, d(x, y)])$. We shall denote by $(1-\beta) x \oplus \beta y$ the unique point $\mathrm{z}$ of $[x, y]$, which satisfies

$$
d(x, z)=\beta d(x, y) \quad \text { and } \quad d(z, y)=(1-\beta) d(x, y) .
$$

Such metric spaces are usually called convex metric spaces [13]. Moreover, if we have

$$
d\left(\frac{1}{2} p \oplus \frac{1}{2} x, \frac{1}{2} p \oplus \frac{1}{2} y\right) \leq \frac{1}{2} d(x, y)
$$

for all $p, x, y$ in $M$, then $M$ is said to be a hyperbolic metric space (see [14]).

Obviously, normed linear spaces are hyperbolic spaces. As nonlinear examples, one can consider the Hadamard manifolds [15], the Hilbert open unit ball equipped with the hyperbolic metric [16], and the CAT(0) spaces [17-19] (see Example 2.1). We will say that a subset $C$ of a hyperbolic metric space $M$ is convex if $[x, y] \subset C$, whenever $x, y$ are in $C$.

Definition 2.1 Let $(M, d)$ be a hyperbolic metric space. We say that $M$ is uniformly convex (in short, UC) if for any $a \in M$, for every $r>0$, and for each $\epsilon>0$

$$
\delta(r, \varepsilon)=\inf \left\{1-\frac{1}{r} d\left(\frac{1}{2} x \oplus \frac{1}{2} y, a\right) ; d(x, a) \leq r, d(y, a) \leq r, d(x, y) \geq r \varepsilon\right\}>0 .
$$

The definition of uniform convexity finds its origin in Banach spaces [20]. To the best of our knowledge, the first attempt to generalize this concept to metric spaces was made in [21]. The reader may also consult $[14,16,22]$.

From now onwards we assume that $M$ is a hyperbolic metric space, and if $(M, d)$ is uniformly convex, then for every $s \geq 0, \epsilon>0$, there exists $\eta(s, \epsilon)>0$ depending on $s$ and $\epsilon$ such that

$$
\delta(r, \varepsilon)>\eta(s, \epsilon)>0 \quad \text { for any } r>s .
$$

Most of the results in this section may be found in [22].

Remark 2.1 [2, 22]

(i) Let us observe that $\delta(r, 0)=0$, and $\delta(r, \varepsilon)$ is an increasing function of $\varepsilon$ for every fixed $r$. 
(ii) For $r_{1} \leq r_{2}$ there holds

$$
1-\frac{r_{2}}{r_{1}}\left(1-\delta\left(r_{2}, \varepsilon \frac{r_{1}}{r_{2}}\right)\right) \leq \delta\left(r_{1}, \varepsilon\right)
$$

(iii) If $(M, d)$ is uniformly convex, then $(M, d)$ is strictly convex, i.e., whenever

$$
d\left(\frac{1}{2} x \oplus \frac{1}{2} y, a\right)=d(x, a)=d(y, a)
$$

for any $x, y, a \in M$, then we must have $x=y$.

Lemma 2.1 $[2,22]$ Assume that $(M, d)$ is uniformly convex. Let $\left\{C_{n}\right\} \subset M$ be a sequence of nonempty, nonincreasing, convex, bounded and closed sets. Let $x \in M$ be such that

$$
0<d=\lim _{n \rightarrow \infty} d\left(x, C_{n}\right)<\infty
$$

Let $x_{n} \in C_{n}$ be such that $d\left(x, x_{n}\right) \rightarrow d$. Then $\left\{x_{n}\right\}$ is a Cauchy sequence.

Recall that a hyperbolic metric space $(M, d)$ is said to have the property $(R)$ if any nonincreasing sequence of nonempty, convex, bounded and closed sets, has a nonempty intersection [23].

Our next result deals with the existence and the uniqueness of the best approximants of convex, closed and bounded sets in a uniformly convex metric space. This result is of interest by itself as uniform convexity implies the property $(R)$, which reduces to reflexivity in the linear case.

Theorem 2.1 [2, 22] Assume that $(M, d)$ is complete and uniformly convex. Let $C \subset M$ be nonempty, convex and closed. Let $x \in M$ be such that $d(x, C)<\infty$. Then there exists a unique best approximant of $x$ in $C$, i.e., there exists a unique $x_{0} \in C$ such that

$$
d\left(x, x_{0}\right)=d(x, C) .
$$

The following result gives the analogue of the well known theorem that states any uniformly convex Banach space is reflexive (see Theorem 2.1 in [16]).

Theorem $2.2[2,22]$ If $(M, d)$ is complete and uniformly convex, then $(M, d)$ has the prop$\operatorname{erty}(R)$.

Note that any hyperbolic metric space $M$, which satisfies the property $(R)$, is complete. The following technical lemma will be needed.

Lemma $2.2[2,22]$ Let $(M, d)$ be uniformly convex. Assume that there exists $R \in[0,+\infty)$ such that

$$
\limsup _{n \rightarrow \infty} d\left(x_{n}, a\right) \leq R, \quad \limsup _{n \rightarrow \infty} d\left(y_{n}, a\right) \leq R, \quad \text { and } \quad \lim _{n \rightarrow \infty} d\left(a, \frac{1}{2} x_{n} \oplus \frac{1}{2} y_{n}\right)=R
$$


Then

$$
\lim _{n \rightarrow \infty} d\left(x_{n}, y_{n}\right)=0
$$

Example 2.1 Let $(X, d)$ be a metric space. A geodesic from $x$ to $y$ in $X$ is a mapping $c$ from a closed interval $[0, l] \subset \mathbb{R}$ to $X$ such that $c(0)=x, c(l)=y$, and $d\left(c(t), c\left(t^{\prime}\right)\right)=\left|t-t^{\prime}\right|$ for all $t, t^{\prime} \in[0, l]$. In particular, $c$ is an isometry and $d(x, y)=l$. The image $\alpha$ of $c$ is called a geodesic (or metric) segment joining $x$ and $y$. The space $(X, d)$ is said to be a geodesic space if every two points of $X$ are joined by a geodesic and $X$ is said to be uniquely geodesic if there is exactly one geodesic joining $x$ and $y$ for each $x, y \in X$, which we will be denoted by $[x, y]$, and called the segment joining $x$ to $y$.

A geodesic triangle $\Delta\left(x_{1}, x_{2}, x_{3}\right)$ in a geodesic metric space $(X, d)$, consisting of three points $x_{1}, x_{2}, x_{3}$ in $X$ (the vertices of $\Delta$ ) and a geodesic segment between each pair of vertices (the edges of $\Delta$ ). A comparison triangle for geodesic triangle $\Delta\left(x_{1}, x_{2}, x_{3}\right)$ in $(X, d)$ is a triangle $\bar{\Delta}\left(x_{1}, x_{2}, x_{3}\right):=\Delta\left(\bar{x}_{1}, \bar{x}_{2}, \bar{x}_{3}\right)$ in $\mathbb{R}^{2}$ such that $d_{\mathbb{R}^{2}}\left(\bar{x}_{i}, \bar{x}_{j}\right)=d\left(x_{i}, x_{j}\right)$ for $i, j \in\{1,2,3\}$. Such a triangle always exists (see [24]).

A geodesic metric space is said to be a $\mathrm{CAT}(0)$ space if all geodesic triangles of appropriate size satisfy the following $\mathrm{CAT}(0)$ comparison axiom:

Let $\Delta$ be a geodesic triangle in $X$, and let $\bar{\Delta} \subset \mathbb{R}^{2}$ be a comparison triangle for $\Delta$. Then $\Delta$ is said to satisfy the CAT(0) inequality if for all $x, y \in \Delta$ and all comparison points $\bar{x}, \bar{y} \in \bar{\Delta}$,

$$
d(x, y) \leq d(\bar{x}, \bar{y}) .
$$

Complete CAT(0) spaces are often called Hadamard spaces (see [18]). If $x, y_{1}, y_{2}$ are points of a $\operatorname{CAT}(0)$ space, and $y_{0}$ is the midpoint of the segment $\left[y_{1}, y_{2}\right]$, which will be denoted by $\frac{y_{1} \oplus y_{2}}{2}$, then the $\mathrm{CAT}(0)$ inequality implies that

$$
d^{2}\left(x, \frac{y_{1} \oplus y_{2}}{2}\right) \leq \frac{1}{2} d^{2}\left(x, y_{1}\right)+\frac{1}{2} d^{2}\left(x, y_{2}\right)-\frac{1}{4} d^{2}\left(y_{1}, y_{2}\right)
$$

This inequality is the $(\mathrm{CN})$ inequality of Bruhat and Tits [25]. As for the Hilbert space, the $(\mathrm{CN})$ inequality implies that $\mathrm{CAT}(0)$ spaces are uniformly convex with

$$
\delta(r, \varepsilon)=1-\sqrt{1-\frac{\varepsilon^{2}}{4}} .
$$

One may also find the modulus of uniform convexity via similar triangles.

Recall that $\tau: M \rightarrow \mathbb{R}_{+}$is called a type if there exists $\left\{x_{n}\right\}$ in $M$ such that

$$
\tau(x)=\limsup _{n \rightarrow \infty} d\left(x, x_{n}\right)
$$

Theorem $2.3[2,22]$ Assume that $(M, d)$ is complete and uniformly convex. Let $C$ be any a nonempty, closed, convex and bounded subset of M. Let $\tau$ be a type defined on C. Then any minimizing sequence of $\tau$ is convergent. Its limit is independent of the minimizing sequence. 


\section{Asymptotic Pointwise Nonexpansive Semigroups}

Recall the definition of an asymptotic pointwise nonexpansive mapping defined in metric spaces $[10,26]$. For similar definition of asymptotic contractions, the reader may consult $[10,11]$.

Definition 3.1 Let $(M, d)$ be a metric space and $C \subset M$ be nonempty and closed. A mapping $T: C \rightarrow C$ is called an asymptotic pointwise mapping if there exists a sequence of mappings $\alpha_{n}: C \rightarrow[0, \infty)$ such that

$$
d\left(T^{n}(x), T^{n}(y)\right) \leq \alpha_{n}(x) d(x, y)
$$

for any $x, y \in C$. If $\limsup _{n \rightarrow \infty} \alpha_{n}(x) \leq 1$ for any $x \in C$, then $T$ is called asymptotic pointwise nonexpansive. A point $x \in C$ is called a fixed point of $T$ if $T(x)=x$. The set of fixed points of $T$ will be denoted by $\operatorname{Fix}(T)$.

This definition is now extended to a one parameter family of mappings.

Definition 3.2 A one-parameter family $\mathcal{F}=\left\{T_{t} ; t \geq 0\right\}$ of mappings from $C$ into itself is said to be an asymptotic pointwise nonexpansive semigroup on $C$ if $\mathcal{F}$ satisfies the following conditions:

(i) $T_{0}(x)=x$ for $x \in C$;

(ii) $T_{t+s}(x)=T_{t}\left(T_{s}(x)\right)$ for $x \in C$ and $t, s \in[0, \infty)$;

(iii) for each $t \geq 0, T_{t}$ is an asymptotic pointwise nonexpansive mapping, i.e., there exists a function $\alpha_{t}: C \rightarrow[0, \infty)$ such that

$$
d\left(T_{t}(x), T_{t}(y)\right) \leq \alpha_{t}(x) d(x, y)
$$

for all $x, y \in C$, such that $\lim _{\sup _{t \rightarrow \infty}} \alpha_{t}(x) \leq 1$ for every $x \in C$, where

$$
\limsup _{t \rightarrow \infty} \alpha_{t}(x)=\inf _{M>0}\left(\sup _{t \geq M} \alpha_{t}(x)\right)
$$

(iv) for each $x \in C$, the mapping $t \rightarrow T_{t}(x)$ is strong continuous.

For each $t \geq 0$, let $\operatorname{Fix}\left(T_{t}\right)$ denote the set of its fixed points. Define then the set of all common fixed points of $\mathcal{F}$ as the following intersection

$$
\operatorname{Fix}(\mathcal{F})=\bigcap_{t \geq 0} \operatorname{Fix}\left(T_{t}\right)
$$

Note that we may assume that $\alpha_{t}(x) \geq 1$ for any $t \geq 0$ and $x \in C$. Indeed set $a_{t}(x)=$ $\max \left(\alpha_{t}(x), 1\right)$. Then one can easily show that

$$
\lim _{t \rightarrow \infty} a_{t}(x)=1 \Longleftrightarrow \limsup _{t \rightarrow \infty} \alpha_{t}(x)=1
$$

Therefore, we will throughout this work assume that $\alpha_{t}(x) \geq 1$, for any $t \geq 0$ and $x \in C$, and $\lim \sup _{t \rightarrow \infty} \alpha_{t}(x)=\lim _{t \rightarrow \infty} \alpha_{t}(x)=1$.

The concept of type functionals is a powerful technical, tool which is used in the proofs of many fixed point results. The definition of a type is based on a given sequence. In this work, we generalize this definition to a one-parameter family of mappings. 
Definition 3.3 Let $(M, d)$ be a hyperbolic metric space. Let $C \subset M$ be convex and bounded. A function $\tau: C \rightarrow[0, \infty]$ is called a (d)-type (or shortly a type) if there exists a one-parameter family $\left\{y_{t}\right\}_{t \geq 0}$ of elements of $C$ such that for any $z \in C$ there holds

$$
\tau(z)=\inf _{M>0}\left(\sup _{t \geq M} d\left(y_{t}, z\right)\right) .
$$

A sequence $\left\{z_{n}\right\} \subset C$ is called a minimizing sequence of $\tau$ if

$$
\lim _{n \rightarrow \infty} \tau\left(z_{n}\right)=\inf \{\tau(z) ; z \in C\} .
$$

A typical method of proof for the fixed point theorems in Banach and metric spaces is to construct a fixed point by finding an element, on which a specific type function attains its minimum. To be able to proceed with this method, one has to know that such an element indeed exists.

The next lemma is the generalization of the minimizing sequence property for types defined by sequences in Lemma 4.3 in [1] to the one-parameter case in modular function spaces.

Lemma 3.1 Assume $(M, d)$ is a uniformly convex hyperbolic metric space. Let $C$ be a nonempty, bounded, closed and convex subset of $M$. Let $\tau$ be a type defined by a oneparameter family $\left\{h_{t}\right\}_{t \geq 0}$ in $C$.

(i) If $\tau\left(z_{1}\right)=\tau\left(z_{2}\right)=\inf _{z \in C} \tau(z)$, then $z_{1}=z_{2}$.

(ii) Moreover any minimizing sequence $\left\{z_{n}\right\}$ of $\tau$ is convergent. Moreover the limit of $\left\{z_{n}\right\}$ is independent of the minimizing sequence.

Proof First let us prove (i). Let $z_{1}, z_{2} \in C$ such that $\tau\left(z_{1}\right)=\tau\left(z_{2}\right)=\inf _{z \in C} \tau(z)$. Assume that $\inf _{z \in C} \tau(z)=0$. Since

$$
d\left(z_{1}, z_{2}\right) \leq d\left(z_{1}, y_{t}\right)+d\left(y_{t}, z_{2}\right)
$$

for any $t \geq 0$, we get

$$
d\left(z_{1}, z_{2}\right) \leq \sup _{t \geq M} d\left(z_{1}, y_{t}\right)+\sup _{t \geq M} d\left(y_{t}, z_{2}\right)
$$

for any $M>0$. Since

$$
\tau(z)=\inf _{M>0}\left(\sup _{t \geq M} d\left(z, y_{t}\right)\right)=\lim _{M \rightarrow \infty} \sup _{t \geq M} d\left(z, y_{t}\right)
$$

for any $z \in C$, we get $d\left(z_{1}, z_{2}\right) \leq \tau\left(z_{1}\right)+\tau\left(z_{2}\right)=0$, which implies $z_{1}=z_{2}$. Therefore, let us assume $\inf _{z \in C} \tau(z)>0$. Assume that $z_{1} \neq z_{2}$. Set

$$
R=\inf _{z \in C} \tau(z) \quad \text { and } \quad \varepsilon=\frac{d\left(z_{1}, z_{2}\right)}{2 R} .
$$

Let $v \in(0, R)$. Then $d\left(z_{1}, z_{2}\right)=2 R \varepsilon \geq(R+v) \varepsilon$. Using the definition of $\tau$, we deduce that there exists $M_{v}>0$ such that

$$
\sup _{t \geq M_{v}} d\left(z_{1}, y_{t}\right) \leq \tau\left(z_{1}\right)+v=R+v \quad \text { and } \quad \sup _{t \geq M_{v}} d\left(z_{2}, y_{t}\right) \leq \tau\left(z_{2}\right)+v=R+v \text {. }
$$


Since $(M, d)$ is uniformly convex, there exists $\eta(R, \varepsilon)>0$ such that

$$
\delta(R+\nu, \varepsilon) \geq \eta(R, \varepsilon)
$$

for any $v \in(0, R)$. So, for any $t \geq M_{\nu}$, we have

$$
d\left(\frac{z_{1} \oplus z_{2}}{2}, y_{t}\right) \leq(R+v)(1-\delta(R+v, \varepsilon)) \leq(R+v)(1-\eta(R, \varepsilon)) .
$$

Hence

$$
\tau\left(\frac{z_{1} \oplus z_{2}}{2}\right) \leq \sup _{t \geq M_{\nu}} d\left(\frac{z_{1} \oplus z_{2}}{2}, y_{t}\right) \leq(R+v)(1-\eta(R, \varepsilon)) .
$$

Since $C$ is convex, we get

$$
R \leq \tau\left(\frac{z_{1} \oplus z_{2}}{2}\right) \leq(R+v)(1-\eta(R, \varepsilon))
$$

If we let $v \rightarrow 0$, we will get

$$
R \leq R(1-\eta(R, \varepsilon))
$$

Contradiction. Therefore, we must have $z_{1}=z_{2}$.

Next, we prove (ii). Set $R=\inf _{z \in C} \tau(z)$. For any $n \geq 1$, set

$$
K_{n}=\overline{\operatorname{conv}}\left\{y_{t} ; t \geq n\right\},
$$

where $\overline{\operatorname{conv}}(A)$ is the intersection of all closed convex subset of $C$, which contains $A \subset C$. Since $C$ is itself closed and convex, we get $K_{n} \subset C$ for any $n \geq 1$. Property $(R)$ will then imply $\bigcap K_{n} \neq \emptyset$. Let $x \in \bigcap K_{n}$. Let $z \in C$ and $\varepsilon>0$. By definition of $\tau(z)$, there exists $M_{\varepsilon}>0$ such that $\sup _{t \geq M_{\varepsilon}} d\left(z, y_{t}\right) \leq \tau(z)+\varepsilon$. Let $n \geq M_{\varepsilon}$. Then for any $t \geq n$, we have $d\left(z, y_{t}\right) \leq$ $\tau(z)+\varepsilon$, i.e., $y_{t} \in B(z, \tau(z)+\varepsilon)$. Since the closed ball $B(z, \tau(z)+\varepsilon)$ is closed and convex, we get $K_{n} \subset B(z, \tau(z)+\varepsilon)$. Hence $x \in B(z, \tau(z)+\varepsilon)$, i.e.,

$$
d(z, x) \leq \tau(z)+\varepsilon
$$

Since $\varepsilon$ was taken arbitrarily greater than 0 , we get $d(z, x) \leq \tau(z)$, for any $z \in C$. Assume that $R=0$. Let $\left\{z_{n}\right\}$ be a minimizing sequence. Then we have $\lim _{n \rightarrow \infty} \tau\left(z_{n}\right)=R=0$. But we just proved that $d\left(z_{n}, x\right) \leq \tau\left(z_{n}\right)$, for any $n \geq 1$. Hence $\left\{z_{n}\right\}$ is convergent to $x$. Note that $x$ is independent of the minimizing sequence. Next, we assume that $R=\inf _{z \in C} \tau(z)>0$. Let $\left\{z_{n}\right\}$ be a minimizing sequence. Assume that $\left\{z_{n}\right\}$ is not Cauchy. For any $n \geq 1$, set

$$
r_{n}=\sup _{i, j \geq n} d\left(z_{i}, z_{j}\right) .
$$

The sequence $\left\{r_{n}\right\}$ is decreasing, and since $\left\{z_{n}\right\}$ is not Cauchy, we get

$$
\inf _{n \geq 1} r_{n}=r>0 .
$$


Set $\varepsilon=\frac{r}{4 R}>0$. Let $v \in(0, R)$. Since $\lim _{n \rightarrow \infty} \tau\left(z_{n}\right)=R$, there exists $n_{0} \geq 1$ such that for any $n \geq 1$, we have $\tau\left(z_{n}\right) \leq R+\frac{v}{2}$. Let $n \geq n_{0}$. Then there exists $i_{n}, j_{n} \geq 1$ such that

$$
d\left(z_{i_{n}}, z_{j_{n}}\right)>r_{n}-\frac{r}{2} \geq \frac{r}{2}=2 R \varepsilon>(R+v) \varepsilon
$$

Using the definition of $\tau$, we deduce the existence of $M>0$ such that

$$
\sup _{t \geq M} d\left(z_{i_{n}}, y_{t}\right) \leq \tau\left(z_{i_{n}}\right)+\frac{v}{2} \leq R+v
$$

and

$$
\sup _{t \geq M} d\left(z_{j_{n}}, y_{t}\right) \leq \tau\left(z_{j_{n}}\right)+\frac{v}{2} \leq R+v
$$

Hence

$$
d\left(\frac{z_{i_{n}} \oplus z_{j_{n}}}{2}, y_{t}\right) \leq(R+v)(1-\delta(R+v, \varepsilon)),
$$

for any $t \geq M$. Since $(M, d)$ is uniformly convex, there exists $\eta(R, \varepsilon)>0$ such that $\delta(R+$ $v, \varepsilon) \geq \eta(R, \varepsilon)$, for any $v>0$. Hence

$$
d\left(\frac{z_{i_{n}} \oplus z_{j_{n}}}{2}, y_{t}\right) \leq(R+v)(1-\eta(R, \varepsilon))
$$

for any $t \geq M$. So

$$
\tau\left(\frac{z_{i_{n}} \oplus z_{j_{n}}}{2}\right) \leq \sup _{t \geq M} d\left(\frac{z_{j_{n}} \oplus z_{j_{n}}}{2}, y_{t}\right) \leq(R+\nu)(1-\eta(R, \varepsilon)) .
$$

Using the definition of $R$, we get

$$
R \leq(R+\nu)(1-\eta(R, \varepsilon))
$$

for any $v \in(0, R)$. If we let $v \rightarrow 0$, we get $R \leq R(1-\eta(R, \varepsilon))$. This contradiction implies that $\left\{z_{n}\right\}$ is Cauchy. Since $M$ is complete, we deduce that $\left\{z_{n}\right\}$ is convergent as claimed. In order to finish the proof of (ii), let us show that the limit of $\left\{z_{n}\right\}$ is independent of the minimizing sequence. Indeed let $\left\{w_{n}\right\}$ be another minimizing sequence of $\tau$. The previous proof will show that $\left\{w_{n}\right\}$ is also convergent. In order to prove that the limits of $\left\{z_{n}\right\}$ and $\left\{w_{n}\right\}$ are equal, let us show that $\lim _{n \rightarrow \infty} d\left(z_{n}, w_{n}\right)=0$. Assume not, i.e., $\lim _{n \rightarrow \infty} d\left(z_{n}, w_{n}\right) \neq$ 0 . Without loss of generality we may assume that there exists $r>0$ such that $d\left(z_{n}, w_{n}\right) \geq r$, for any $n \geq 1$. Set $\varepsilon=\frac{r}{2 R}>0$. Let $v \in(0, R)$. Since $\lim _{n \rightarrow \infty} \tau\left(z_{n}\right)=\lim _{n \rightarrow \infty} \tau\left(w_{n}\right)=R$, there exists $n_{0} \geq 1$ such that for any $n \geq n_{0}$, we have $\tau\left(z_{n}\right) \leq R+\frac{\nu}{2}$, and $\tau\left(w_{n}\right) \leq R+\frac{\nu}{2}$. Fix $n \geq n_{0}$. Then

$$
d\left(z_{n}, w_{n}\right) \geq r=2 R \varepsilon>(R+v) \varepsilon .
$$

Using the definition of $\tau$, we deduce the existence of $M>0$ such that

$$
\sup _{t \geq M} d\left(z_{n}, y_{t}\right) \leq \tau\left(z_{n}\right)+\frac{v}{2} \leq R+v,
$$


and

$$
\sup _{t \geq M} d\left(w_{n}, y_{t}\right) \leq \tau\left(w_{n}\right)+\frac{v}{2} \leq R+v
$$

Hence

$$
d\left(\frac{z_{n} \oplus w_{n}}{2}, y_{t}\right) \leq(R+v)(1-\delta(R+v, \varepsilon))
$$

for any $t \geq M$. Since $(M, d)$ is uniformly convex, there exists $\eta(R, \varepsilon)>0$ such that $\delta(R+$ $v, \varepsilon) \geq \eta(R, \varepsilon)$ for any $v>0$. Hence

$$
d\left(\frac{z_{n} \oplus w_{n}}{2}, y_{t}\right) \leq(R+v)(1-\eta(R, \varepsilon))
$$

for any $t \geq M$. So

$$
\tau\left(\frac{z_{n} \oplus w_{n}}{2}\right) \leq \sup _{t \geq M} d\left(\frac{z_{n} \oplus w_{n}}{2}, y_{t}\right) \leq(R+\nu)(1-\eta(R, \varepsilon))
$$

Using the definition of $R$, we get

$$
R \leq(R+\nu)(1-\eta(R, \varepsilon))
$$

for any $v \in(0, R)$. If we let $v \rightarrow 0$, we get $R \leq R(1-\eta(R, \varepsilon))$. This contradiction implies that $\lim _{n \rightarrow \infty} d\left(z_{n}, w_{n}\right)=0$, which completes the proof.

\section{Main result}

Using the Lemma 3.1, we are ready to prove the main fixed point result for asymptotic pointwise nonexpansive semigroup in metric spaces.

Theorem 4.1 Let $(M, d)$ be a uniformly convex metric space. Let $C$ be a closed bounded convex nonempty subset of $M$. Let $\mathcal{F}=\left\{T_{t} ; t \geq 0\right\}$ be an asymptotically pointwise nonexpansive semigroup on $C$. Then $\mathcal{F}$ has a common fixed point and the set $\operatorname{Fix}(\mathcal{F})$ of common fixed points is closed and convex.

Proof Let us fix $x \in C$ and define the type function $\tau$ on $C$ by

$$
\tau(z)=\inf _{M>0}\left(\sup _{t \geq M} d\left(T_{t}(x), z\right)\right)
$$

Since $C$ is bounded, we get $\tau(z)<+\infty$, for any $z \in C$. Hence $\tau_{0}=\inf \{\tau(z) ; z \in C\}$ exists. For any $n \geq 1$, there exists $z_{n} \in C$, such that

$$
\tau_{0} \leq \tau\left(z_{n}\right)<\tau_{0}+\frac{1}{n}
$$

Therefore, $\lim _{n \rightarrow \infty} \tau\left(z_{n}\right)=\tau_{0}$, i.e., $\left\{z_{n}\right\}$ is a minimizing sequence for $\tau$. By using Lemma 3.1, there exists $z \in C$ such that $\left\{z_{n}\right\}$ converges to $z$. Let us now prove that $z \in \operatorname{Fix}(\mathcal{F})$. Note 
that

$$
d\left(T_{s+t}(x), T_{s}(w)\right) \leq \alpha_{s}(w) d\left(T_{t}(x), w\right)
$$

for $s, t>0$ and $w \in C$. Using the definition of $\tau$, we get

$$
\tau\left(T_{s}(w)\right) \leq \sup _{t+s \geq M} d\left(T_{s+t}(x), T_{s}(w)\right) \leq \alpha_{s}(w) \sup _{t \geq M-s} d\left(T_{t}(x), w\right)
$$

for any $M>s$, which implies that

$$
\tau\left(T_{s}(w)\right) \leq \alpha_{s}(h) \tau(w)
$$

Since $\lim \sup _{s \rightarrow \infty} \alpha_{s}\left(z_{1}\right) \leq 1$, there exists $s_{1}>0$ such that for any $s \geq s_{1}$, we have $\alpha_{s}\left(z_{1}\right)<$ $1+1$. Repeating this argument, one will find $s_{2}>s_{1}+1$ such that for any $s \geq s_{2}$, we have $\alpha_{s}\left(z_{2}\right)<1+\frac{1}{2}$. By induction, we will construct a sequence $\left\{s_{n}\right\}$ of positive numbers such that $s_{n+1}<s_{n}+\frac{1}{n}$, and for any $s \geq s_{n}$, we have $\alpha_{s}\left(z_{n}\right)<1+\frac{1}{n}$. Let us fix $t \geq 0$. Then the inequality (4.1) will imply that

$$
\tau\left(T_{s_{n}+t}\left(z_{n}\right)\right) \leq \alpha_{s_{n}+t}\left(z_{n}\right) \tau\left(z_{n}\right) \leq\left(1+\frac{1}{n}\right) \tau\left(z_{n}\right)
$$

for any $n \geq 1$. In particular we get $\left\{T_{s_{n}+t}\left(z_{n}\right)\right\}$ is a minimizing sequence of $\tau$. Therefore, the technical Lemma 3.1 will imply that $\left\{T_{s_{n}+t}\left(z_{n}\right)\right\}$ converges to $z$, for any $t \geq 0$. In particular, $\left\{T_{S_{n}}\left(z_{n}\right)\right\}$ converges to $z$. Since

$$
d\left(T_{s_{n}+t}\left(z_{n}\right), T_{t}(z)\right) \leq \alpha_{t}(z) d\left(T_{s_{n}}\left(z_{n}\right), z\right)
$$

we get $\left\{T_{s_{n}+t}\left(z_{n}\right)\right\}$ converges to $T_{t}(z)$. Finally, using

$$
d\left(T_{t}(z), z\right) \leq d\left(T_{t}(z), T_{s_{n}+t}\left(z_{n}\right)\right)+d\left(T_{s_{n}+t}\left(z_{n}\right), z\right)
$$

we get $T_{t}(z)=z$. Since $t$ was arbitrarily positive, we get $z \in \operatorname{Fix}(\mathcal{F})$, i.e., $\operatorname{Fix}(\mathcal{F})$ is nonempty. Next, let us prove that $\operatorname{Fix}(\mathcal{F})$ is closed. Let $\left\{z_{n}\right\}$ be in $\operatorname{Fix}(\mathcal{F})$, which converges to $z$. Since

$$
d\left(T_{s}\left(z_{n}\right), T_{s}(z)\right) \leq \alpha_{s}(z) d\left(z_{n}, z\right)
$$

for any $n \geq 1$ and $s>0$, we get $\left\{T_{s}\left(z_{n}\right)\right\}$ is convergent, and its limit is $T_{s}(z)$. Since $z_{n} \in$ $\operatorname{Fix}(\mathcal{F})$, we get $\left\{T_{s}\left(z_{n}\right)\right\}=\left\{z_{n}\right\}$. In other words, $\left\{z_{n}\right\}$ converges to $T_{s}(z)$ and $z$. The uniqueness of the limit, will then imply $T_{s}(z)=z$, for any $s \geq 0$, i.e., $z \in \operatorname{Fix}(\mathcal{F})$. Therefore, $\operatorname{Fix}(\mathcal{F})$ is closed. Let us finish the proof of Theorem 4.1 by showing that $\operatorname{Fix}(\mathcal{F})$ is convex. It is sufficient to show that

$$
z=\frac{z_{1} \oplus z_{2}}{2} \in \operatorname{Fix}(\mathcal{F})
$$

for any $z_{1}, z_{2} \in \operatorname{Fix}(\mathcal{F})$. Without loss of generality, we assume that $z_{1} \neq z_{2}$. Let $s>0$. We have

$$
d\left(z_{1}, T_{s}(z)\right)=d\left(T_{s}\left(z_{1}\right), T_{s}(z)\right) \leq \alpha_{s}(z) d\left(z_{1}, z\right)
$$


and

$$
d\left(z_{2}, T_{s}(z)\right)=d\left(T_{s}\left(z_{2}\right), T_{s}(z)\right) \leq \alpha_{s}(z) d\left(z_{2}, z\right) .
$$

Since $d\left(z_{1}, z\right)=d\left(z_{2}, z\right)=\frac{d\left(z_{1}, z_{2}\right)}{2}$, and

$$
d\left(z_{1}, z_{2}\right) \leq d\left(z_{1}, T_{s}(z)\right)+d\left(z_{2}, T_{s}(z)\right) \leq \alpha_{s}(z) d\left(z_{1}, z_{2}\right),
$$

we conclude that

$$
\lim _{s \rightarrow \infty} d\left(z_{1}, T_{s}(z)\right)=\lim _{s \rightarrow \infty} d\left(z_{2}, T_{s}(z)\right)=\frac{d\left(z_{1}, z_{2}\right)}{2} .
$$

Similarly, we have

$$
d\left(z_{1}, \frac{z \oplus T_{s}(z)}{2}\right) \leq \frac{1}{2} d\left(z_{1}, z\right)+\frac{1}{2} d\left(z_{1}, T_{s}(z)\right),
$$

and

$$
d\left(z_{2}, \frac{z \oplus T_{s}(z)}{2}\right) \leq \frac{1}{2} d\left(z_{2}, z\right)+\frac{1}{2} d\left(z_{2}, T_{s}(z)\right) .
$$

Since

$$
d\left(z_{1}, z_{2}\right) \leq d\left(z_{1}, \frac{z \oplus T_{s}(z)}{2}\right)+d\left(z_{2}, \frac{z \oplus T_{s}(z)}{2}\right),
$$

we conclude that

$$
\lim _{s \rightarrow \infty} d\left(z_{1}, \frac{z \oplus T_{s}(z)}{2}\right)=\lim _{s \rightarrow \infty} d\left(z_{2}, \frac{z \oplus T_{s}(z)}{2}\right)=\frac{d\left(z_{1}, z_{2}\right)}{2} .
$$

Therefore, we have

$$
\lim _{s \rightarrow \infty} d\left(z_{1}, T_{s}(z)\right)=\lim _{s \rightarrow \infty} d\left(z_{1}, \frac{z \oplus T_{s}(z)}{2}\right)=\frac{d\left(z_{1}, z_{2}\right)}{2} .
$$

Lemma 2.2 will then imply that

$$
\lim _{s \rightarrow \infty} d\left(z, T_{s}(z)\right)=0 .
$$

Hence $\lim _{s \rightarrow \infty} d\left(z, T_{s+t}(z)\right)=0$ for any $t \geq 0$. Since

$$
d\left(T_{t}(z), T_{s+t}(z)\right) \leq \alpha_{t}(z) d\left(z, T_{s}(z)\right),
$$

we get $\lim _{s \rightarrow \infty} d\left(T_{t}(z), T_{s+t}(z)\right)=0$. Finally, using the inequality

$$
d\left(z, T_{t}(z)\right) \leq d\left(z, T_{s+t}(z)\right)+d\left(T_{t}(z), T_{s+t}(z)\right),
$$

and letting $s \rightarrow \infty$, we get $T_{t}(z)=z$ for any $t \geq 0$, i.e., $z \in \operatorname{Fix}(\mathcal{F})$. 


\section{Competing interests}

The authors declare that they have no competing interests.

\section{Authors' contributions}

All authors participated in the design of this work and performed equally. All authors read and approved the final manuscript.

\section{Author details}

${ }^{1}$ Department of Mathematics, King Abdulaziz University, P.O. Box 80203, Jeddah, 21593, Saudi Arabia. ${ }^{2}$ Department of Mathematical Sciences, University of Texas at El Paso, El Paso, Texas, USA. ${ }^{3}$ Department of Mathematics and Statistics, King Fahd University of Petroleum \& Minerals, Dhahran, 31261, Saudi Arabia.

Received: 15 February 2013 Accepted: 18 June 2013 Published: 28 August 2013

\section{References}

1. Khamsi, MA, Kozlowski, WK: On asymptotic pointwise contractions in modular function spaces. Nonlinear Anal. 73 2957-2967 (2010)

2. Khamsi, MA, Kozlowski, WK: On asymptotic pointwise nonexpansive mappings in modular function spaces. J. Math Anal. Appl. 380, 697-708 (2011)

3. Belluce, LP, Kirk, WA: Fixed-point theorems for families of contraction mappings. Pac. J. Math. 18, $213-217$ (1966)

4. Belluce, LP, Kirk, WA: Nonexpansive mappings and fixed-points in Banach spaces. III. J. Math. 11, 474-479 (1967)

5. Browder, FE: Nonexpansive nonlinear operators in a Banach space. Proc. Natl. Acad. Sci. USA 54, 1041-1044 (1965)

6. Bruck, RE: A common fixed point theorem for a commuting family of nonexpansive mappings. Pac. J. Math. 53, 59-71 (1974)

7. DeMarr, RE: Common fixed-points for commuting contraction mappings. Pac. J. Math. 13, 1139-1141 (1963)

8. Lim, TC: A fixed point theorem for families of nonexpansive mappings. Pac. J. Math. 53, 487-493 (1974)

9. Tan, K-K, Xu, H-K: An ergodic theorem for nonlinear semigroups of Lipschitzian mappings in Banach spaces. Nonlinear Anal. 19(9), 805-813 (1992)

10. Kirk, WA, Xu, HK: Asymptotic pointwise contractions. Nonlinear Anal. 69, 4706-4712 (2008)

11. Hussain, N, Khamsi, MA: On asymptotic pointwise contractions in metric spaces. Nonlinear Anal. 71(10), 4423-4429 (2009)

12. Khamsi, MA: Nonlinear semigroups in modular function spaces. Math. Jpn. 379, 1-9 (1992)

13. Menger, K: Untersuchungen über allgemeine Metrik. Math. Ann. 100, 75-163 (1928)

14. Reich, S, Shafrir, I: Nonexpansive iterations in hyperbolic spaces. Nonlinear Anal. 15, 537-558 (1990)

15. Busemann, H: Spaces with non-positive curvature. Acta Math. 80, 259-310 (1948)

16. Goebel, K, Reich, S: Uniform Convexity, Hyperbolic Geometry, and Nonexpansive Mappings. Series of Monographs and Textbooks in Pure and Applied Mathematics, vol. 83. Dekker, New York (1984)

17. Kirk, WA: Fixed Point Theory for Nonexpansive Mappings, I and II. Lecture Notes in Mathematics, vol. 886, pp. 485-505. Springer, Berlin (1981)

18. Kirk, WA: A fixed point theorem in CAT(0) spaces and R-trees. Fixed Point Theory Appl. 2004(4), $309-316$ (2004)

19. Leustean, L: A quadratic rate of asymptotic regularity for CAT(0)-spaces. J. Math. Anal. Appl. 325, 386-399 (2007)

20. Clarkson, JA: Uniformly convex spaces. Trans. Am. Math. Soc. 40(3), 396-414 (1936)

21. Goebel, K, Sekowski, T, Stachura, A: Uniform convexity of the hyperbolic metric and fixed points of holomorphic mappings in the Hilbert ball. Nonlinear Anal. 4, 1011-1021 (1980)

22. Khamsi, MA, Khan, AR: Inequalities in metric spaces with applications. Nonlinear Anal. TMA 74, 4036-4045 (2011)

23. Khamsi, MA: On metric spaces with uniform normal structure. Proc. Am. Math. Soc. 106, 723-726 (1989)

24. Bridson, M, Haefliger, A: Metric Spaces of Non-Positive Curvature. Springer, Berlin (1999)

25. Bruhat, F, Tits, J: Groupes réductifs sur un corps local. I. Données radicielles valuées. Publ. Math. IHÉS 41, 5-251 (1972)

26. Khamsi, MA: On asymptotically nonexpansive mappings in hyperconvex metric spaces. Proc. Am. Math. Soc. 132 365-373 (2004)

doi:10.1186/1687-1812-2013-230

Cite this article as: Al-Mezel and Khamsi: Fixed point of asymptotic pointwise nonexpansive semigroups in metric spaces. Fixed Point Theory and Applications 2013 2013:230.

\section{Submit your manuscript to a SpringerOpen ${ }^{\circ}$ journal and benefit from:}

- Convenient online submission

- Rigorous peer review

- Immediate publication on acceptance

- Open access: articles freely available online

- High visibility within the field

- Retaining the copyright to your article 